\title{
Nasopharyngeal bacterial carriage in young children in Greenland: a population at high risk of respiratory infections
}

\author{
J. E. NAVNE ${ }^{1}$, M. L. BØRRESEN ${ }^{1,2} *$ H. C. SLOTVED ${ }^{3}$, M. ANDERSSON ${ }^{1}$, \\ M. MELBYE ${ }^{1}$, K. LADEFOGED ${ }^{4}$ AND A. $\mathrm{KOCH}^{1}$ \\ ${ }^{1}$ Department of Epidemiology Research, Statens Serum Institut, Copenhagen, Denmark \\ ${ }^{2}$ Department of Pediatrics, Rigshospitalet, Copenhagen, Denmark \\ ${ }^{3}$ Department of Microbiology and Infection Control, Statens Serum Institut, Copenhagen, Denmark \\ ${ }^{4}$ Department of Internal Medicine, Queen Ingrid's Hospital, Nuuk, Greenland
}

Received 20 March 2016; Final revision 12 May 2016; Accepted 16 June 2016; first published online 13 July 2016

\section{SUMMARY}

The incidence of childhood respiratory infections in Greenland is among the highest globally. We performed a population-based study of 352 Greenlandic children aged 0-6 years aiming to describe rates and risk factors for carriage of four key bacteria associated with respiratory infections, their antimicrobial susceptibility and inter-bacterial associations. Nasopharyngeal swabs were tested for Streptococcus pneumoniae grouped by serotypes included (VT) or not included (NVT) in the 13-valent pneumococcal conjugate vaccine, non-typable Haemophilus influenzae (NTHi), Staphylococcus aureus and Moraxella catarrhalis. S. pneumoniae was detected from age 2 weeks with a peak carriage rate of $60 \%$ in 2-year-olds. Young age and having siblings attending a daycare institution were associated with pneumococcal carriage. Overall co-colonization with $\geqslant 2$ of the studied bacteria was $52 \%$. NTHi showed a positive association with NVT pneumococci and $M$. catarrhalis, respectively, M. catarrhalis was positively associated with $S$. pneumoniae, particular VT pneumococci, whereas $S$. aureus were negatively associated with NTHi and $M$. catarrhalis. Nasopharyngeal bacterial carriage was present unusually early in life and with frequent co-colonization. Domestic crowding increased odds of carriage. Due to important bacterial associations we suggest future surveillance of pneumococcal conjugate vaccine's impact on carriage in Greenland to also include other pathogens.

Key words: Children, Greenland, Inuit, nasopharyngeal carriage, PCV-13, pneumococci, risk factors.

\section{INTRODUCTION}

Respiratory infections are a major healthcare problem in the Inuit population of the Arctic. In Greenland [1], where almost $90 \%$ of the population is of Inuit origin,

\footnotetext{
* Author for correspondence: Dr M. L. Børresen, Department of Epidemiology Research, Statens Serum Institut, Artillerivej 5, 2300 Copenhagen, Denmark.

(Email: mlb@ssi.dk)
}

the incidence of clinically verified upper and lower respiratory infections in children aged $0-2$ years is among the highest in the world [1]. In particular, otitis media is highly prevalent, characterized by early onset, recurrent episodes progressing to chronic otitis media with long-term consequences such as hearing loss and impaired language acquisition [2]. The burden of disease is almost exclusively carried by the Inuit population, whereas non-Inuit in Greenland have lower risks of respiratory infections [1]. 
In addition, the incidence of invasive pneumococcal disease (IPD) is markedly high in native populations of the Arctic including Greenlandic Inuit with significantly higher mortality than in non-Inuit [3]. However, reasons for this ethnic health disparity and the high incidence of respiratory tract infections are basically unknown. In other populations, genetic as well as environmental factors including socioeconomic conditions have been shown to influence the risk of respiratory infections [3]. Except for use of childcare centres, passive smoking, and a parental history of recurrent infections [1], particular risk factors for respiratory tract infections have not been identified in the Inuit.

Worldwide, some of the most clinically relevant bacteria involved in childhood respiratory and invasive bacterial diseases include Streptococcus pneumoniae, non-typable Haemophilus influenzae, Moraxella catarrhalis, and Staphylococcus aureus [4, 5]. The bacteria colonize the nasopharynx except for $S$. aureus which resides in the anterior nasal cavity [6]. Nasopharyngeal carriage, which is highly frequent in young children, is considered the essential first step in the development of respiratory and invasive bacterial infections. Although asymptomatic in themselves, the bacteria may migrate to cause either local infections such as otitis media, sinusitis and pneumonia or systemic potentially life-threatening invasive diseases such as bacteraemia and meningitis [7]. Nasopharyngeal carriage may thus be considered an infectious reservoir for bacterial autoinfections as well as for transmission to other individuals in the community $[7,8]$.

Factors known to influence bacterial carriage rates, besides age, are: bacterial and viral intra- and interspecies interactions (commensal and pathogenic), function of the immune system, and environmental factors [7].

In late 2010 the 13 -valent pneumococcal conjugate vaccine Prevenar $13^{\circledR}$ (PCV-13; Pfizer, USA) was introduced in the Greenlandic childhood vaccination programme at ages 3 and 5 months with a booster at age 12 months. The impact of PCV-13 introduction in Greenland is unknown. Its predecessor, the 7-valent pneumococcal conjugate vaccine (PCV-7), which was never used in Greenland, has in other settings been shown not only to reduce incidence of IPD caused by pneumococcal serotypes included in the vaccine (also called vaccine serotypes; VT), but also to prevent nasopharyngeal carriage by VT $[9,10]$. However, invasive disease caused by non-vaccine pneumococcal serotypes (NVT) and other bacteria (so-called 'replacement disease') may have emerged as a consequence of the vacant nasopharyngeal niche being refilled by non-vaccine serotype pneumococci or other respiratory pathogens [9-16]. In 2000, Alaska introduced the pneumococcal vaccine into the childhood vaccination programme (PCV-7). Significant pneumococcal serotype shifts were observed, particularly in the Native Alaskan Inuit population, with reduced prevalence of VT-IPD but also one of the highest reported degrees of IPD caused by NVT and a total incidence of IPD reaching the pre-PCV7 vaccination level [11].

In this cross-sectional population-based study of Greenlandic children aged 0-6 years, we aimed to determine prevalence and risk factors for nasopharyngeal carriage by $S$. pneumoniae, non-typable $H$. influenzae (NTHi), M. catarrhalis and S. aureus, including antibiotic susceptibility testing and patterns of bacterial associations to provide surveillance data during the introduction of PCV-13 vaccine in Greenland.

\section{METHODS AND MATERIALS}

\section{Population/study design}

Greenland is the world's largest island with more than three quarters covered by ice and a population of 56370 (2013) persons living in towns and settlements scattered along the coastline. Approximately $88 \%$ of the people are Inuit and the rest mainly Caucasians (Danes) [17].

Two towns and nearby settlements were chosen as the study area. Tasiilaq, one of two towns on the East coast with 1800 inhabitants and 800 persons living in three settlements, and Sisimiut on the West coast, the second-largest town of Greenland with 5460 inhabitants and 350 persons in one settlement.

Since 1972, all citizens of Greenland have been given a unique identification number registered in the Civil Registration System (CRS). The daily updated CRS contains vital information on place and date of birth, gender, birth order, siblings, parents, current and earlier addresses [18], and the unique personal identification number allows for accurate linkage between other national registers. We identified all children aged 0 to $<7$ years in October 2011 in the CRS and their parents living in the study area and invited them to participate. After written and oral informed consents were obtained from parents/caregivers, a questionnaire was completed regarding 
number of siblings, daycare institution attendance, breastfeeding (at any given period and if so duration and if currently breastfeeding), recent antibiotic use (within the last 3 months), domestic tobacco exposure, recent respiratory tract infections (within the last 3 months), hospitalizations (at any given period), number of rooms, number of people sleeping in the same room, in-house water supply and heating source. Data on PCV-13 vaccination status was obtained through nationwide medical files. The study was conducted during autumn 2011, from October to December.

\section{Nasopharyngeal sampling}

The standard procedure for nasopharyngeal sampling recommended by World Health Organization in 2003 was used [19]. Skim milk-tryptone-glucose-glycerin medium (STGG) has proven useful for the study of respiratory pathogens [20]. A nasopharyngeal swab sample was taken using minitip flocked nylon swabs (FLOQSwabs ${ }^{\mathrm{TM}}$, Copan, Italy) inserted via the nasal cavity into the posterior wall of the nasopharynx, rotated $180^{\circ}$ then placed in $1 \mathrm{ml}$ STGG medium and stored at $20^{\circ} \mathrm{C}$ for a maximum of 3 weeks before being transported by air at $-20^{\circ} \mathrm{C}$ to Statens Serum Institut (SSI), Copenhagen, Denmark, for storage at $-80^{\circ} \mathrm{C}$.

\section{Laboratory analysis}

After thawing, the specimens were vortexed and $50 \mu 1$ of each sample was streaked onto a $5 \%$ horse blood agar, a chocolate agar and an antibiotic chocolate agar plate (SSI). S. pneumoniae, S. aureus and NTHi were primarily targeted on blood agar medium. The cross-streaking with a strain of $S$. aureus on the blood agar was done to identify NTHi since the two species grow in symbiosis. M. catarrhalis was primarily isolated from the chocolate agar plates. The antibiotic chocolate agar targeted anaerobic species such as Neisseria menigitidis, containing four different antibiotics, polymyxin B (27 $246 \mathrm{IE})$, lincomycin (0.001 g), amphotericin B (0.002 g) and trimethoprim $(0.003 \mathrm{~g})$ to inhibit the growth of most Gram-positive and Gram-negative bacteria as well as fungal species. To increase the likelihood of detecting low-density carriage and carriage of multiple pneumococcal serotypes, we also added $50 \mu \mathrm{l}$ of the nasopharyngeal sample to a $2 \mathrm{ml}$ serum ox broth and incubated in $\mathrm{CO}_{2}, 37^{\circ} \mathrm{C}$ for $24 \mathrm{~h}$, before plating again as described above. This method has proven efficient in increasing the detection level of nasopharyngeal pneumococci [21].

Bacterial identification was based on colony morphology as ascertained by conventional microbiological procedures and verified by MALDI/TOF mass spectrometry [22]. All isolates were tested for antimicrobial susceptibility using the disk diffusion test and EUCAST breakpoints [23]. Pneumococci were identified based on $\alpha$-haemolysis, optochin sensitivity and capsular reaction (known as 'Quellung'). Non-typable pneumococci were identified using the bile solubility test.

Pneumococcal group determination was performed directly on the serum broth-enriched nasopharyngeal samples by Pneumotest Latex ${ }^{\circledR}$ agglutination (SSI, Denmark). Serotypes were identified by Quellung [24] reaction with the use of type-specific antisera from SSI $[21,25]$.

\section{Statistical analyses}

The use of serum broth enrichment increased the detection of $S$. pneumoniae, NTHi and S. aureus, whereas detection of $M$. catarrhalis was reduced when using this enrichment. Due to these selective growth advantages, we decided to base the analysis of carriage rates on either a positive original or serum broth-enriched nasopharyngeal sample. However, since serum broth addition changed the bacterial composition, the analyses of risk-factor and bacterial associations were based solely on results from the original swab samples.

Risk-factor analyses and tests for inter-bacterial associations were done by logistic regression analysis (PROC LOGISTIC; SAS v. 9.3, USA) with bacterial colonization as outcome. Each exposure variable was first tested separately in a univariable model and only if significant at the 5\% level included in a multivariable model. Based on this, the final model was adjusted for age, sex, ethnicity and PCV-13 status (i.e. having received $\geqslant 1$ dose of PCV-13 or not). Pneumococcal serotypes were grouped in vaccine types (VT), i.e. serotypes included in the PCV-13 vaccine and non-vaccine types (NVT), not included in the PCV-13 vaccine, and treated as separate groups of bacteria in the analyses.

\section{Ethics}

The authors assert that all procedures contributing to this work comply with the ethical standards of the relevant national and institutional committees on human experimentation and with the Helsinki Declaration of 1975, as revised in 2008. The study was 
approved by the Greenlandic Scientific Commission (Journal no. 2011-056257, doc. no. 738293) and the Danish Data Protection Agency (2008-54-0427).

\section{RESULTS}

A total of 450 children were invited and 352 (78\%) children aged $0-6$ years [median $2 \cdot 8$ years, interquartile range (IQR) $1 \cdot 1-4 \cdot 8$ years] consented and were enrolled. The majority $(92 \%)$ was of Inuit origin and $52 \%$ were males (Table 1 ). Approximately one third of the children had received $\geqslant 1$ dose of PCV-13 vaccination $(13 \%$ one dose, $16 \%$ two doses, $8 \%$ three doses). The median age of children who had received $\geqslant 1$ dose of $\mathrm{PCV}-13$ was 1.2 years (IQR $0.6-1.7$ years) and the median age of unvaccinated children was $4 \cdot 4$ years (IQR 3-5.6 years).

The serum broth enrichment increased the detection of low-density growth, in particular S. pneumoniae, NTHi and $S$. aureus, whereas the detection of $M$. catarrhalis was reduced (Table 2).

\section{Carriage of potentially pathogenic bacteria}

Overall, $293(83 \%)$ children carried one or more potentially pathogenic bacteria (Fig. 1a). Carriage was established at an early age with $85 \%$ of infants aged $<2$ months being colonized, rising to $100 \%$ in children aged 2-4 months. Carriage rates fluctuated between $85 \%$ and $100 \%$ during the first year (Fig. 1a). Thereafter, a steady decline to reach an overall carriage rate of $70 \%$ in 6-year-olds was observed (Fig. 1b).

\section{M. catarrhalis}

The most frequent colonizing bacteria was $M$. catarrhalis with an overall carriage rate of 53\% (188 isolates). Rates increased during the first year and peaked in those aged $1-2$ years $(70 \%)$, and then decreased to $20 \%$ in preschool children. All isolates were resistant to penicillin and ampicillin, intermediately resistant to cephalosporins (cefuroxime) and fully susceptible to macrolides.

\section{S. pneumoniae}

A total of 185 S. pneumoniae isolates were detected in 178 children, due to co-colonization with multiple serotypes in $4 \%$ of participants, resulting in an overall carriage rate of $50 \%$, with the highest carriage rate before age 3 years $(60 \%)$ levelling off to $45 \%$ in subsequent years (Fig. $1 b)$.
Table 1. Demographic characteristics of 352 healthy Greenlandic children aged 0-6 years, 2011, Tasiilaq and Sisimiut, Greenland

\begin{tabular}{|c|c|}
\hline Category & $\begin{array}{l}\text { Total }(N=352) \\
n(\%)\end{array}$ \\
\hline \multicolumn{2}{|l|}{ Age (years) } \\
\hline 0 to $<2$ & $134(38)$ \\
\hline 2 to $<4$ & $93(26)$ \\
\hline 4 to $<7$ & $125(36)$ \\
\hline \multicolumn{2}{|l|}{ Sex } \\
\hline Female & $169(48)$ \\
\hline Male & $181(52)$ \\
\hline \multicolumn{2}{|l|}{ Ethnicity } \\
\hline Inuit & $324(92)$ \\
\hline Mixed & $11(3)$ \\
\hline Other & $17(5)$ \\
\hline \multicolumn{2}{|c|}{ PCV-13 vaccinated* } \\
\hline Yes & $130(37)$ \\
\hline No & $222(63)$ \\
\hline \multicolumn{2}{|c|}{ Geographical region in Greenland $\dagger$} \\
\hline East coast & $122(35)$ \\
\hline West coast & $230(65)$ \\
\hline \multicolumn{2}{|c|}{ Daycare centre attendance } \\
\hline Yes & $258(73)$ \\
\hline No & $84(24)$ \\
\hline Missing & $10(3)$ \\
\hline \multicolumn{2}{|c|}{ Current breastfeeding } \\
\hline Yes & $131(37)$ \\
\hline No & $210(60)$ \\
\hline Missing & $11(3)$ \\
\hline \multicolumn{2}{|l|}{ Ever breastfed } \\
\hline Yes & $220(63)$ \\
\hline No & $98(28)$ \\
\hline Missing & $34(9)$ \\
\hline \multicolumn{2}{|c|}{ Having siblings attend a daycare centre $\S$} \\
\hline Yes & $137(39)$ \\
\hline No & $201(57)$ \\
\hline Missing & $14(4)$ \\
\hline \multicolumn{2}{|c|}{ Number of persons per room in household\| } \\
\hline 0 to $<2$ & $251(71)$ \\
\hline$\geqslant 2$ & $79(22)$ \\
\hline Missing & $22(7)$ \\
\hline \multicolumn{2}{|c|}{ Exposure to tobacco smoke } \\
\hline Yes & $83(24)$ \\
\hline No & $269(76)$ \\
\hline Missing & $6(2)$ \\
\hline
\end{tabular}

*PCV-13: Having received $\geqslant 1$ dose of the 13-valent pneumococcal conjugate vaccine or not.

$\dagger$ Geographical site: East Greenland (Tasilaq) and West Greenland (Sisimiut).

¥ Daycare attendance: Current attendance at a daycare centre. $\S$ Sibling in daycare: Having siblings attend a daycare institution. \| Persons per room: Number of persons per household divided by number of rooms exclusive of kitchen, entrance hall and bathroom.

ITobacco exposure: When one or both parents/caregivers smoke. 
Table 2. Overall nasopharyngeal carriage rates in 352 healthy Greenlandic children aged 0-6 years. Results listed according to positive culture from either the original nasopharyngeal samples, the serum broth-enriched samples, positive in samples either with or without serum broth enrichment and finally positive in both types of samples

\begin{tabular}{lllcllc}
\hline \hline Type of swab sample & S. pneumoniae & NTHi & M. catarrhalis & S. aureus & Others* & Sterile \\
\hline Original & $137(39)$ & $108(30)$ & $177(50)$ & $22(6 \cdot 8)$ & $109(31)$ & $27(7 \cdot 5)$ \\
Serum broth enriched & $175(50)$ & $141(40)$ & $88(25)$ & $39(11)$ & $101(28)$ & $8(2)$ \\
Original or serum broth & $176(50)$ & $152(43)$ & $188(53)$ & $41(11 \cdot 6)$ & $151(43)$ & $32(9)$ \\
Original and serum broth & $136(37)$ & $97(27)$ & $77(22)$ & $20(5 \cdot 6)$ & $59(17)$ & $3(1)$ \\
\hline \hline
\end{tabular}

NTHi, non-typable Haemophilus influenza.

Values given are $n(\%)$.

* Others: Moraxella species in particular nonliquefaciens, coagulase-negative staphylococci, non-haemolytic streptococci, Haemophilus influenzae types $\mathrm{B}, \mathrm{E}$ and $\mathrm{F}(n=5,1 \%)$ and haemolytic streptococci groups $\mathrm{A}, \mathrm{B}$ and $\mathrm{G}(n=9,2 \cdot 5 \%)$.
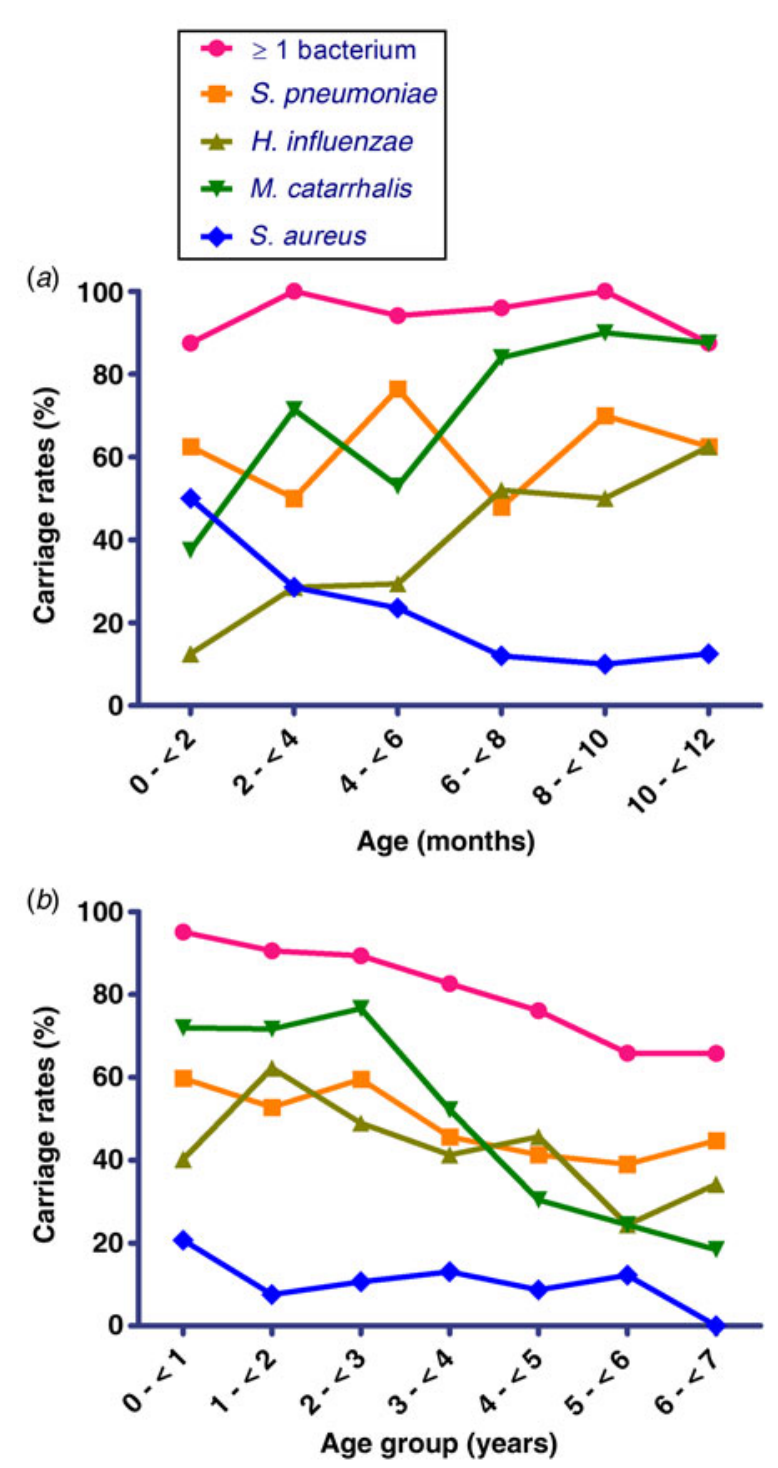

Fig. 1. (a) Nasopharyngeal bacterial carriage in healthy Greenlandic children aged $(a)$ 0-12 months, $(b)$ 0-6 years.
Carriage was detected as early as age 2 weeks, and by age 2 months $50 \%$ of children carried pneumococci (Fig. $1 a$ ). The most frequently carried serotypes were $6 \mathrm{~B}, 6 \mathrm{C}, 15 \mathrm{~B}$, $16 \mathrm{~F}, 19 \mathrm{~A}, 22 \mathrm{~F}, 23 \mathrm{~F}, 33 \mathrm{~F}$ and $35 \mathrm{~F}$ accounting for $78 \%$ of all isolates. Of these, only serotypes $6 \mathrm{~B}$ and $19 \mathrm{~A}$ are included in the PCV-13 vaccine. All S. pneumoniae isolates were fully susceptible to penicillin (oxacillin) except one isolate of type $6 \mathrm{C}$ that also showed resistance to erythromycin and clindamycin.

\section{NTHi}

Overall carriage was $43 \%$ (152 isolates), with increasing rates during the first 2 years from $10 \%$ to $60 \%$. After peaking in 2-year-olds rates dropped to $35 \%$ in children aged 5-6 years. We only identified three capsular H. influenzae isolates (types B and E). Of NTHi isolates, $36 \%$ were penicillin-resistant and $\beta$-lactamaseproducing and thus resistant to ampicillin, amoxicillin and piperacillin according to EUCAST breakpoints v. 3.1 [23]. Five percent of NTHi isolates were resistant to trimethoprim-sulfamethoxazole.

\section{S. aureus}

A different carriage pattern was observed for $S$. aureus with highest rates in children aged $0-1$ month $(50 \%)$, followed by a decline to around $10 \%$ in 1-year-olds where it stabilized until age 6-7 years. A total of 41 $(11 \cdot 6 \%)$ isolates were identified. The majority $(90 \%)$ of $S$. aureus isolates were penicillin-resistant but susceptible to dicloxacillin.

\section{Risk factors for carriage}

For all pathogens young age was significantly associated with increased bacterial carriage (Table 3). 
Table 3. Risk factors for carriage of S. pneumoniae, non-typable Haemophilus influenzae, M. catarrhalis, S. aureus or $\geqslant 1$ of either bacteria in Greenlandic children aged 0 to $<7$ years in Tasiilaq and Sisimiut 2011, adjusted for age, sex, ethnicity and PCV-13 status

\begin{tabular}{|c|c|c|c|c|c|c|c|c|c|c|c|}
\hline \multirow[b]{2}{*}{ Variable } & \multirow[b]{2}{*}{$N(\%)$} & \multicolumn{2}{|l|}{ S. pneumoniae } & \multicolumn{2}{|l|}{ NTHi } & \multicolumn{2}{|l|}{ M. catarrhalis } & \multicolumn{2}{|l|}{ S. aureus } & \multicolumn{2}{|l|}{$\geqslant 1$ bacteria } \\
\hline & & OR $(95 \% \mathrm{CI})$ & $P$ & OR $(95 \% \mathrm{CI})$ & $P$ & OR $(95 \% \mathrm{CI})$ & $P$ & OR $(95 \% \mathrm{CI})$ & $P$ & OR $(95 \% \mathrm{CI})$ & $P$ \\
\hline Age (years) & $352(100)$ & $0 \cdot 8(0 \cdot 69-0 \cdot 9)$ & $<0 \cdot 01$ & $0.83(0 \cdot 7-0.9)$ & $<0 \cdot 01$ & $0 \cdot 65(0 \cdot 5-0 \cdot 7)$ & $<0.01$ & $0 \cdot 7(0 \cdot 6-0 \cdot 9)$ & $0 \cdot 04$ & $0 \cdot 6(0 \cdot 5-0 \cdot 7)$ & $<0 \cdot 01$ \\
\hline \multicolumn{12}{|l|}{ Sex } \\
\hline Female & $169(49)$ & 1 (ref.) & & 1 (ref.) & & 1 (ref.) & & 1 (ref.) & & 1 (ref.) & \\
\hline Male & $183(51)$ & $1 \cdot 3(0 \cdot 8-2 \cdot 0)$ & $0 \cdot 26$ & $1 \cdot 0(0 \cdot 6-1 \cdot 7)$ & $0 \cdot 78$ & $1 \cdot 5(0 \cdot 9-2 \cdot 4)$ & $0 \cdot 06$ & $0 \cdot 9(0 \cdot 2-5 \cdot 2)$ & $0 \cdot 97$ & $1 \cdot 4(0 \cdot 9-2 \cdot 4)$ & $0 \cdot 14$ \\
\hline \multicolumn{12}{|l|}{ Ethnicicty } \\
\hline Inuit & $324(92)$ & 1 (ref.) & & 1 (ref.) & & 1 (ref.) & & 1 (ref.) & & 1 (ref.) & \\
\hline Mixed/other & $28(8)$ & $0 \cdot 7(0 \cdot 3-1 \cdot 5)$ & 0.32 & $1 \cdot 6(0 \cdot 7-3 \cdot 5)$ & 0.29 & $0 \cdot 5(0 \cdot 2-1 \cdot 2)$ & $0 \cdot 12$ & $1 \cdot 1(0 \cdot 2-5 \cdot 2)$ & $0 \cdot 88$ & $0 \cdot 6(0 \cdot 2-1 \cdot 5)$ & $0 \cdot 30$ \\
\hline \multicolumn{12}{|l|}{ PCV-13* } \\
\hline No doses & $222(63)$ & 1 (ref.) & & 1 (ref.) & & 1 (ref.) & & 1 (ref.) & & 1 (ref.) & \\
\hline$\geqslant 1$ dose & $130(37)$ & $0 \cdot 5(0 \cdot 3-1 \cdot 1)$ & $0 \cdot 07$ & $1 \cdot 0(0 \cdot 6-2 \cdot 0)$ & 0.77 & $1 \cdot 1(0 \cdot 6-2 \cdot 1)$ & $0 \cdot 73$ & $0 \cdot 2(0 \cdot 1-0 \cdot 7)$ & $0 \cdot 01$ & $0 \cdot 7(0 \cdot 3-1 \cdot 5)$ & 0.37 \\
\hline \multicolumn{12}{|l|}{ Region $\dagger$} \\
\hline West & $230(65)$ & 1 (ref.) & & 1 (ref.) & & 1 (ref.) & & 1 (ref.) & & 1 (ref.) & \\
\hline East & $122(35)$ & $0 \cdot 9(0 \cdot 6-1 \cdot 6)$ & $0 \cdot 94$ & $0 \cdot 4(0 \cdot 2-0 \cdot 7)$ & $<0 \cdot 01$ & $0 \cdot 4(0 \cdot 3-0 \cdot 8)$ & $<0 \cdot 01$ & $1 \cdot 1(0 \cdot 4-3 \cdot 1)$ & $0 \cdot 83$ & $0.6(0 \cdot 3-0.9)$ & $0 \cdot 04$ \\
\hline \multicolumn{12}{|l|}{ Daycaret } \\
\hline No & $84(24)$ & 1 (ref.) & & 1 (ref.) & & 1 (ref.) & & 1 (ref.) & & 1 (ref.) & \\
\hline Yes & $258(73)$ & $1 \cdot 0(0 \cdot 5-1 \cdot 9)$ & $0 \cdot 96$ & $4 \cdot 7(2 \cdot 3-9 \cdot 6)$ & $<0 \cdot 01$ & $2 \cdot 4(1 \cdot 3-4 \cdot 7)$ & $0 \cdot 02$ & $0 \cdot 4(0 \cdot 1-1 \cdot 6)$ & $0 \cdot 50$ & $1 \cdot 5(0 \cdot 7-3 \cdot 1)$ & 0.31 \\
\hline \multicolumn{12}{|c|}{ Siblings in daycare§ } \\
\hline No & $201(57)$ & 1 (ref.) & & 1 (ref.) & & 1 (ref.) & & 1 (ref.) & & 1 (ref.) & \\
\hline Yes & $137(39)$ & $1 \cdot 6(1 \cdot 1-2 \cdot 2)$ & $0 \cdot 04$ & $1 \cdot 7(1 \cdot 1-2 \cdot 4)$ & $0 \cdot 04$ & $1 \cdot 1(0 \cdot 7-1 \cdot 9)$ & $0 \cdot 68$ & $0 \cdot 6(0 \cdot 2-1 \cdot 6)$ & $0 \cdot 28$ & $1 \cdot 1(0 \cdot 6-1 \cdot 9)$ & 0.62 \\
\hline \multicolumn{12}{|l|}{ Persons/room\| } \\
\hline$<2$ & $251(72)$ & 1 (ref.) & & 1 (ref.) & & 1 (ref.) & & 1 (ref.) & & 1 (ref.) & \\
\hline$\geqslant 2$ & $79(28)$ & $1 \cdot 7(1.0-2.9)$ & $0 \cdot 05$ & $1 \cdot 2(0 \cdot 7-2 \cdot 1)$ & $0 \cdot 55$ & $0 \cdot 9(0 \cdot 5-1 \cdot 7)$ & $0 \cdot 95$ & $0 \cdot 6(0 \cdot 2-2 \cdot 3)$ & $0 \cdot 49$ & $0 \cdot 9(0 \cdot 5-1 \cdot 8)$ & 0.95 \\
\hline \multicolumn{12}{|c|}{ Tobacco exposure } \\
\hline No & $83(24)$ & 1 (ref.) & & 1 (ref.) & & 1 (ref.) & & 1 (ref.) & & 1 (ref.) & \\
\hline Yes & $269(76)$ & $1 \cdot 3(0 \cdot 7-2 \cdot 2)$ & 0.39 & $1 \cdot 6(0 \cdot 8-2 \cdot 9)$ & $0 \cdot 15$ & $1 \cdot 9(1 \cdot 1-3 \cdot 5)$ & $0 \cdot 03$ & $0 \cdot 3(0 \cdot 1-0 \cdot 8)$ & $0 \cdot 01$ & $1 \cdot 1(0 \cdot 6-2 \cdot 0)$ & 0.79 \\
\hline \multicolumn{12}{|c|}{ OR, Odds ratio; CI, confidence interval; NTHi, non-typable Haemophilus influenzae; PCV-13 status, 13 -valent pneumococcal conjugate vaccine. } \\
\hline
\end{tabular}


Table 4. Odds of co-colonization between pneumococcal vaccine (VT) or non-vaccine (NVT) serotypes, non-typable $\mathrm{H}$. influenzae (NTHi), M. catarrhalis and $\mathrm{S}$. aureus in 352 Greenlandic children aged $<7$ years. Analyses are based on isolates from original swab samples without serum broth enrichment and with no restrictions regarding other potential co-colonizing bacteria. OR are adjusted for age, sex, ethnicity and PCV-13 status

\begin{tabular}{|c|c|c|c|c|c|c|c|c|c|c|c|c|}
\hline \multirow[b]{2}{*}{ Bacterium } & \multicolumn{3}{|c|}{$\mathrm{VT}^{*}$} & \multicolumn{3}{|c|}{ NTHi } & \multicolumn{3}{|c|}{ M. catarrhalis } & \multicolumn{3}{|c|}{ S. aureus } \\
\hline & $n$ & $\mathrm{aOR}$ & $P$ & $n$ & $\mathrm{aOR}$ & $P$ & $n$ & $\mathrm{aOR}$ & $P$ & $n$ & $\mathrm{aOR}$ & $P$ \\
\hline \multicolumn{13}{|l|}{$\mathrm{NVT}^{\dagger}$} \\
\hline No & 113 & 1 (ref.) & & 34 & 1 (ref.) & & 85 & 1 (ref.) & & 105 & & \\
\hline Yes & 4 & $0 \cdot 15(0 \cdot 1-0 \cdot 3)$ & $<0 \cdot 01$ & 41 & $2 \cdot 3(1 \cdot 4-3 \cdot 7)$ & $<0 \cdot 01$ & 32 & $1.6(0.95-2.5)$ & 0.08 & 12 & $0.75(0.35-1 \cdot 4)$ & $0 \cdot 44$ \\
\hline \multicolumn{13}{|c|}{ - } \\
\hline Yes & & & & 42 & 1 (ref.) & & 42 & 1 (ref.) & & 50 & 1 (ref.) & \\
\hline No & & & & 18 & $0.7(0 \cdot 4-1 \cdot 5)$ & $0 \cdot 40$ & 18 & $3.4(1 \cdot 7-6.5)$ & $<0 \cdot 01$ & 10 & $1 \cdot 2(0 \cdot 4-3 \cdot 9)$ & 0.68 \\
\hline \multicolumn{13}{|l|}{ NTHi } \\
\hline No & & & & & & & 46 & 1 (ref.) & & 46 & 1 (ref.) & \\
\hline Yes & & & & & & & 37 & $4 \cdot 5(2 \cdot 6-7 \cdot 8)$ & $<0.01$ & 37 & $0.2(0 \cdot 1-0.9)$ & 0.04 \\
\hline \multicolumn{13}{|c|}{ M. catarrhalis } \\
\hline No & & & & & & & & & & 64 & 1 (ref.) & \\
\hline Yes & & & & & & & & & & 6 & $0 \cdot 1(0 \cdot 02-0 \cdot 4)$ & $<0 \cdot 01$ \\
\hline
\end{tabular}

aOR, Adjusted odds ratio; NTHi: non-typable Haemophilus influenza.

*VT, Pneumococcal serotypes included in the 13-valent pneumococcal conjugate vaccine (PCV-13).

$\dagger$ NVT, Pneumococcal serotypes not included in PCV-13 vaccine.

Significant findings $(P<0.05)$ in bold.

Crowding-related factors such as attending a daycare institution, having a sibling in daycare or living with $\geqslant 2$ persons per room, increased the odds of bacterial carriage (except for $S$. aureus). Furthermore, we found significantly higher carriage rates of NTHi and M. catarrhalis in children from Sisimiut compared to children from Tasiilaq even when adjusting for daycare attendance. Ethnicity did not show a clear association with carriage. Neither did breastfeeding, recent antibiotic use, recent respiratory tract infections, hospitalizations, in-house water supply and heating source (data not shown).

\section{Bacterial associations}

Co-colonization between any two or more of the studied bacteria appeared in $52 \%$ of children, primarily in young infants, and most frequently between $S$. pneumoniae and $M$. catarrhalis (33\%). NTHi showed a positive association with NVT pneumococci and $M$. catarrhalis, respectively, but a negative association with $S$. aureus (Table 4). M. catarrhalis was positively associated with $S$. pneumoniae, particular VT pneumococci, and negatively associated with $S$. aureus. When restricting the pairwise interaction analysis to individuals with no concurrent colonization by any other of the studied bacteria none of the estimates changed in direction, but some interactions were less pronounced. However, the association between NTHi and M. catarrhalis, remained positive (odds ratio $4 \cdot 0,95 \%$ confidence interval $1 \cdot 3-11 \cdot 7)$.

\section{DISCUSSION}

Overall, we found nasopharyngeal bacterial colonization in Greenlandic children to occur unusually early in life, with frequent bacterial co-colonization and continuing high carriage rates in older children. Furthermore, we found crowding-related risk factors for bacterial carriage and indications of clinically important associations between four key pathogens frequently related to respiratory and invasive infections in young children.

Pneumococcal carriage was acquired within the first 2 weeks of life and followed by a prevalence of about $60 \%$ during the next 2 years. In a systematic review of nasopharyngeal carriage in children, Adegbola et al. found that pneumococcal carriage rates in children from low- and lower-middle-income countries generally were higher (up to $93 \%$ ) compared to middle- and high-income countries where the highest reported carriage rates were 58\% [26]. High carriage rates of pneumococci have been associated with a high prevalence of respiratory infections [27]. Greenland has one 
of the highest incidences of respiratory tract infections [28]. Furthermore, invasive pneumococcal disease is frequent in Inuit in the Arctic [3] and S. pneumoniae is the most frequent cause of invasive bacterial diseases in Greenland, associated with high morbidity and mortality [29]. Yet, unlike most other high-risk populations with very high rates of bacterial carriage [30-32], this population-based study shows carriage rates comparable to those of paediatric populations from Europe and the United States with much lower incidences of respiratory tract infections [27, 33]. In other words, the carriage rate in itself does not seem to be the most significant feature for the high disease burden in Greenlandic children. However, we observed that carriage in our population occurred very early, i.e. at age 2 weeks, compared to a mean age of 6 months for first acquisition in paediatric populations from Europe and the United States [7]. Furthermore, we saw a high rate of co-colonization in our population, which is likely to have implications for polymicrobial respiratory infections. These findings correspond to findings in other high-risk groups, although relatively few studies have described the combined carriage of respiratory pathogens. In a carriage study of Australian Aboriginals aged 0-2 years, who share the same very high disease burden from otitis media as the Greenlandic Inuit, they found higher carriage rates of respiratory pathogens and more frequent carriage by multiple pathogens in Aboriginal children compared to non-Aboriginals and colonization in Aboriginals began at an earlier age. The numbers were compatible with our findings [30]. Furthermore, in the Australian study as well as in the present study, the overall carriage rates were only moderately reduced with age as opposed to lowrisk populations where the rates after a peak incidence in 2-year-olds decreases gradually [8]. This pattern of early age at first acquisition and ongoing polymicrobial colonization through infancy and childhood may account for the high disease burden in this Inuit population.

To our knowledge, previous studies on bacterial nasopharyngeal carriage in the Inuit population, have almost exclusively focused on pneumococcal carriage without including other potential pathogenic bacteria [34, 35]. Only a single Greenlandic study from 1993 has described nasopharyngeal bacterial and viral co-carriage in young children with acute otitis media and healthy controls. However, in that study, few risk factors for carriage of specific bacteria were identified, except for young age and current acute otitis media that were associated with increased S. pneumoniae carriage rates [36].

We consistently found that young age increased the odds of bacterial carriage regardless of the studied species. This association has partly been attributed to close contacts and high transmission rates between young children combined with poorly developed immunity in this age group. In line with other studies [37] breastfeeding did not seem to protect against bacterial carriage. Breastfeeding has, however, been shown to lower the risk of otitis media and invasive pneumococcal disease [27, 38]. This could indicate, that higher levels of serotype-specific serum $\operatorname{IgG}$ than what is achievable from breastfeeding may be required to prevent or clear colonization. As suspected, crowding-related factors increased the risk of carriage. Attending a daycare institution is quite common in Greenland with $73 \%$ of the children in this study doing so. Furthermore, the average number of persons per household is higher in Greenland (3.4 in towns and $4 \cdot 4$ in settlements) than in, for example, Denmark (overall 2.1) [17]. Ethnicity in itself did not show any association with carriage suggesting that environmental factors play the most critical role for the risk of nasopharyngeal carriage in this population. However, the number of non-Inuit was low. Hence, the study may be underpowered for conclusions regarding ethnic associations. This study was conducted during autumn. The effect of seasonality on nasopharyngeal bacterial carriage is inconsistent and seems to vary according to the study population. Midwinter has been associated with an increased rate of carriage for reasons that remain unclear, but it may be related to closer interpersonal contacts due to more indoor activity. However, in other studies the effect of season is absent [7]. Homøe et al.'s [36] study on nasopharyngeal carriage in Greenlandic children did not study potential seasonal variation on rates of carriage, but Koch et al. found no seasonal pattern of the overall incidence of acute respiratory infections [28] during a 2-year cohort study of Inuit children living in Greenland.

Odds for NTHi carriage were substantially increased if NVT pneumococci or $M$. catarrhalis were also detected. Diverging results have been reported on co-colonization with these pathogens [39]. Xu et al., who studied both healthy children and children with an upper respiratory infection noted that bacterial interactions differed in healthy and symptomatic children. This may indicate that inter-bacterial dynamics are influenced by infections [40]. 
The positive association between NTHi and NVT pneumococci, but not VT, may point towards a serotype-specific association between NTHi and pneumococcal serotypes. Spijkerman et al. [14] found persistently higher prevalence rates of NTHi carriage and pneumococcal non-PCV-7 types in young asymptomatic children 3 and $4 \frac{1}{2}$ years after PCV-7 vaccination together with an almost complete eradication of PCV-7 type pneumococci.

The Finnish Otitis Media trials found an increase in the proportion of otitis media caused by NTHi following $\mathrm{PCV}-7$ vaccination compared to the pre-PCV-7 era [41]. This raises the question if increased NTHi carriage rates can be expected after widespread use of PCV-13 in Greenland due to the inter-bacterial interaction and the anticipated serotype shift from VT- to NVT-colonizing pneumococci. Theoretically, that may put PCV-13-immunized children at a higher risk of otitis media caused by NTHi.

This study has some limitations. Since we only recruited children from two areas of Greenland we cannot be certain that they are representative of the whole country. Moreover, the study was conducted during the first year after the introduction of the PCV-13 vaccine and thus the observed carriage rates may to some extent be confounded by this vaccine introduction. However, this effect is likely to be limited for various reasons. First, only $37 \%$ of the children had received $\geqslant 1$ PCV-13 vaccination, second only $8 \%$ of the children were fully vaccinated with three doses, and finally vaccination only occurred in the youngest children. The inter-bacterial associations we have observed may be temporary during the introduction of PCV-13. Whether these associations persist may be clarified by a follow-up study that recently has been carried out in the same study area. Finally, the association models between two species may be simplistic and in fact not reflect the complexity of events occurring within the nasopharyngeal flora, and thus only be revealed in studies using more complex methods, such as microbiome studies.

In conclusion, we found the overall nasopharyngeal carriage rates in healthy children in Greenland to be comparable with low-risk paediatric populations, although colonization begins very early in life, with frequent bacterial co-colonization, and ongoing high carriage rates of four clinically relevant bacteria. Our results also indicate that colonization does not occur at random but may be affected by important inter-bacterial associations.

\section{ACKNOWLEDGEMENTS}

The authors give special thanks for the help and hospitality provided by the chief medical doctor at the Hospital of Tasiilaq, Hans-Christian Florian Sørensen and at the Hospital of Sisimiut, Ove Rosing Olsen, as well as the staff at the hospitals helping with the logistics. The fieldwork would not have been successful without the extensive help from the interpreters Susanne Vid Stein and Antoinette Kuitse. A huge effort was made to finish the analysis of nasopharyngeal samples in time by the technical laboratory staff at the Statens Serum Institut, with special thanks to Kirsten Olsson, Kirsten Burmeister and Monja Hammer as well as medical student Jacqueline Mistry. We are sincerely grateful to the many participating children and their families for spending their time with us and giving us the opportunity to fulfil the study. Finally, we are very appreciative of the friendly help from the public schools and daycare institutions of Sisimiut and Tasiilaq.

The study received funding from The Commission for Scientific Research in Greenland, co-financed by The Danish Research Council (grant no. 100905576); The A. P. Møller Foundation for the Advancement of Medical Science; and the Aase \& Ejnar Danielsens Foundation.

\section{DECLARATION OF INTEREST}

H. C. Slotved participates in a research project supported by Pfizer and declares no conflict of interest regarding the present study. None of the other authors have any conflict of interest.

\section{REFERENCES}

1. Koch A, et al. Risk factors for acute respiratory tract infections in young Greenlandic children. American Journal of Epidemiology 2003; 158: 374-384.

2. Jensen RG, et al. Long-term follow-up of chronic suppurative otitis media in a high-risk children cohort. International Journal of Pediatric Otorhinolaryngology 2011; 75: 948-954.

3. Bruce MG, et al. International Circumpolar Surveillance System for invasive pneumococcal disease, 1999-2005. Emerging Infectious Diseases 2008; 14: 25-33.

4. O'Brien KL, et al. Burden of disease caused by Streptococcus pneumoniae in children younger than 5 years: global estimates. Lancet 2009; 374: 893-902.

5. Poll T Van Der, Opal SM, Van der Poll T. Pathogenesis, treatment, and prevention of pneumococcal pneumonia. Lancet 2009; 374: 1543-1556. 
6. Sivaraman K, Venkataraman N, Cole AM. Staphylococcus aureus nasal carriage and its contributing factors. Future Microbiology 2009; 4: 999-1008.

7. Garcia-Rodriguez JA, Fresnadillo Martinez MJ. Dynamics of nasopharyngeal colonization by potential respiratory pathogens. Journal of Antimicrobial Chemotherapy 2002; 50 (Suppl. S): 59-73.

8. Simell B, et al. The fundamental link between pneumococcal carriage and disease. Expert Review of Vaccines 2012; 11: 841-855.

9. O'Brien KL, et al. Effect of pneumococcal conjugate vaccine on nasopharyngeal colonization among immunized and unimmunized children in a communityrandomized trial. Journal of Infectious Diseases 2007; 196: $1211-1220$.

10. Scott JR, et al. Impact of more than a decade of pneumococcal conjugate vaccine use on carriage and invasive potential in Native American communities. Journal of Infectious Diseases 2012; 205: 280-288.

11. Singleton RJ, et al. Invasive pneumococcal disease caused by nonvaccine serotypes among alaska native children with high levels of 7-valent pneumococcal conjugate vaccine coverage. Journal of the American Medical Association 2007; 297: 1784-1792.

12. Feikin DR, et al. Serotype-specific changes in invasive pneumococcal disease after pneumococcal conjugate vaccine introduction: a pooled analysis of multiple surveillance sites. PLoS Medicine 2013; 10:e1001517.

13. Taylor S, et al. Impact of pneumococcal conjugate vaccination on otitis media: a systematic review. Clinical Infectious Diseases 2012; 54:1765-1773.

14. Spijkerman J, et al. Long-term effects of pneumococcal conjugate vaccine on nasopharyngeal carriage of $S$. pneumoniae, S. aureus, $H$. influenzae and $M$. catarrhalis. PLoS ONE 2012; 7: e39730.

15. Jokinen J, Palmu AA, Kilpi T. Acute otitis media replacement and recurrence in the Finnish otitis media vaccine trial. Clinical Infectious Diseases 2012; 55:1673-1676.

16. Bogaert D, et al. Colonisation by Streptococcus pneumoniae and Staphylococcus aureus in healthy children. Lancet. 2004; 363: 1871-1882.

17. Greenland in Figures Greenland. Kalaallit Nunaat, 2013 (http://www.stat.gl/dialog/main.asp?lang=da\&version= 2013\&link $=$ GF\&subthemecode $=$ p1\&colcode $=$ p. .

18. Pedersen CB, et al. The Danish Civil Registration System. A cohort of eight million persons. Danish Medical Bulletin 2006; 53: 441-449.

19. O'Brien KL, Nohynek H. Report from a WHO Working Group: standard method for detecting upper respiratory carriage of Streptococcus pneumoniae. Pediatric Infectious Disease Journal 2003; 22:e1-11.

20. Kaijalainen T, et al. Survival of Streptococcus pneumoniae, Haemophilus influenzae, and Moraxella catarrhalis frozen in skim milk-tryptone-glucose-glycerol medium. Journal of Clinical Microbiology 2004; 42:412-414.

21. Kaltoft MS, et al. An easy method for detection of nasopharyngeal carriage of multiple Streptococcus pneumoniae serotypes. Journal of Microbiology Methods 2008; 75:540-544.
22. Carbonnelle E, et al. MALDI-TOF mass spectrometry tools for bacterial identification in clinical microbiology laboratory. Clinical Biochemistry 2011; 44:104 109.

23. European Committee on Antimicrobial Susceptibility Testing. Breakpoint tables for interpretation of MICs and zone diameters. Version 3.1, 2013 (http://www. eucast.org.).

24. Merrill CW, et al. Rapid identification of pneumococci. Gram stain vs. the quellung reaction. New England Journal of Medicine 1973; 288:510-2.

25. Slotved H-C, et al. Simple, rapid latex agglutination test for serotyping of pneumococci (PneumotestLatex). Journal of Clinical Microbiology 2004; 42: 2518-22.

26. Adegbola R A, et al. Carriage of Streptococcus pneumoniae and other respiratory bacterial pathogens in low and lower-middle income countries: a systematic review and meta-analysis. PLOS ONE 2014; 9:e103293.

27. Garcia-Rodriguez JA. Dynamics of nasopharyngeal colonization by potential respiratory pathogens. Journal of Antimicrobial Chemotherapy 2002; 50: 59-74.

28. Koch A, et al. Population-based study of acute respiratory infections in children, Greenland. Emerging Infectious Diseases 2002; 8: 586-593.

29. Meyer A, et al. Population-based survey of invasive bacterial diseases, Greenland, 1995-2004. Emerging Infectious Diseases 2008; 14: 76-79.

30. Mackenzie G A, et al. Epidemiology of nasopharyngeal carriage of respiratory bacterial pathogens in children and adults: cross-sectional surveys in a population with high rates of pneumococcal disease. $B M C$ Infectious Diseases 2010; 10: 304.

31. Dunne EM, et al. Effect of pneumococcal vaccination on nasopharyngeal carriage of Streptococcus pneumoniae, Haemophilus influenzae, Moraxella catarrhalis, and Staphylococcus aureus in Fijian children. Journal of Clinical Microbiology 2012; 50: 1034-1048.

32. Van Den Biggelaar AHJ, et al. Effect of early carriage of Streptococcus pneumoniae on the development of pneumococcal protein-specific cellular immune responses in infancy. Pediatric Infectious Disease Journal 2012; 31: 243-258.

33. Harboe ZB, et al. A pneumococcal carriage study in danish pre-school children before the introduction of pneumococcal conjugate vaccination. Open Microbiology Journal 2012; 6: 40-54.

34. Moore MR, et al. Impact of a conjugate vaccine on community-wide carriage of nonsusceptible Streptococcus pneumoniae in Alaska. Journal of Infectious Diseases 2004; 190: 2031-2038.

35. Hammitt LL, et al. Indirect effect of conjugate vaccine on adult carriage of Streptococcus pneumoniae: an explanation of trends in invasive pneumococcal disease. Journal of Infectious Diseases 2006; 193: 1487 1494.

36. Homøe $\mathbf{P}$, et al. High rate of nasopharyngeal carriage of potential pathogens among children in Greenland: results of a clinical survey of middle-ear disease. Clinical Infectious Diseases 1996; 23: 1081-1090. 
37. Principi N, et al. Risk factors for carriage of respiratory pathogens in the nasopharynx of healthy children. Ascanius Project Collaborative Group. Pediatric Infectious Disease Journal 1999; 18: 517-523.

38. Levine OS, et al. Risk factors for invasive pneumococcal disease in children: a population-based casecontrol study in North America. Pediatrics 1999; 103: E28.
39. Dunne EM, et al. Nasopharyngeal microbial interactions in the era of pneumococcal conjugate vaccination. Vaccine 2013; 31: 2333-2342.

40. Xu Q, et al. Nasopharyngeal bacterial interactions in children. Emerging Infectious Diseases 2012; 18: 1738-1745.

41. Eskola J, et al. Efficacy of a pneumococcal conjugate vaccine against acute otitis media. New England Journal of Medicine 2001; 344: 403-409. 